\title{
Water Ecological Compensation Methods for Provincial water function zone
}

\author{
Luo Huihuang ${ }^{1, a}$, Zhao Shanshan ${ }^{2, b}$ and Peng Wenqi ${ }^{3, c}$ \\ ${ }^{1}$ China Institute of Water Resources and Hydropower, Beijing, China \\ ${ }^{2}$ Hebei Agricultural University, Baoding, China \\ ${ }^{3}$ China Institute of Water Resources and Hydropower, Beijing, China

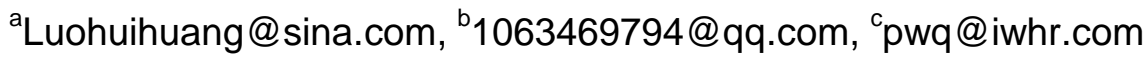

\begin{abstract}
Keywords: water ecological compensation; water quality; provincial water function zone.
Abstract. The paper studies on ecological compensation method for provincial water environmental function zone. The authors analyze the water quality of water environment function zone, identify the characteristic provincial water quality issues. The authors propose water ecological compensation mechanism for the functional areas of provincial water restrictions pollutant management, water features in phases and coordinate inter-provincial water standards ribbon pollution control contradictory provide technical support.
\end{abstract}

\section{Introduction}

The water environmental issues become serious with the rapidly economic, social development. The provincial water function zone which connect upstream and downstream district zone is very important for interprovincial environment. The water quality deterioration of interprovincial water function zone, will result in economic and social conflicts. Therefore, improvement of water quality for interprovincial water function zone is particularly important. Research on water ecological compensation methods for provincial water function zone has important significance.

Many scholars have carried out research for water ecological compensation. Li Xiaoyan[1] concluded on basin water ecological compensation connotation. Qiao Xuning[2] analyzed the research status at home and abroad river basin ecological compensation and existing problems. Zhang Bin[3] analyzed theoretical water ecological compensation mechanism. Liang Yan[4] putted forward connotation basin water ecological compensation mechanism and the establishment of ecological compensation mechanism basin water issues to be addressed. Li Jinxiu[5], who referred to our country and across administrative regions upstream and downstream water pollution increasingly sharp disputes is one of the characteristics of pollution of water resources in the protection of economic compensation countermeasures. Xu Jian[6] discussed the theoretical basis of regional water ecological compensation and xin'an river Basin. Fu Yicheng[7] discussed cross-regional water basin ecological compensation standard calculation method. Li Jinxiu[8] analyzed the major functional areas of water quality factor, functional area water quality summary investigate excessive risk. Previous studies from different levels of water-regional ecological compensation mechanism were analyzed and discussed, but the combination of research provincial water function zone water quality standards are rare. This paper proposes interprovincial water function zone water ecological compensation methods, by analyzing of water quality problems and their classification characteristic provincial water function zone.

\section{Provincial Water Quality Status and Problems}

\section{Interprovincial water quality}

According to the 2011 National Water Resources Bulletin[9], the annual water function zone water quality compliance rate was $46.4 \%$, the provincial boundary function zone compliance rate less than $39.1 \%$. The provincial boundary function zone of Songhua river's water quality was poor, the water quality standard of $30.8 \%$ function zone was IV, 3.8\% was V. In Liaohe river, 33.3\% was IV, 16.7\% 
worse than $V$ [10]. In Haihe river, $2.5 \%$ was $I V \sim V$, inferior $V$ water accounted for $56.5 \%$ [11]. Yellow river $16.2 \%$ was IV class, $10.8 \%$ was V, $27.0 \%$ worse than V [12]. In Huaihe River, water quality compliance rate was accounted for $27.5 \%, 23.5 \%$ for flood period, $33.3 \%$ for non-flood period[13]. In Yangtze River, all year 22.2\%worse than III [14]. In taihu Lake basin, 25.7\% worse than III [15]. In Pearl river, 22.5\% worse than IV V, 2.5\% worse than V [16].

The main water quality index which become worse for each river basin are total phosphorus, ammonia nitrogen, chemical oxygen demand, permanganate index, BOD, fluoride, volatile phenol, petroleum, fecal coliform and biochemical need five days oxygen and so on. The most important project exceeded chemical oxygen demand, permanganate index, ammonia nitrogen.

\section{Interprovincial water function zone water quality issues}

Many provincial water function zones which water quality is poor locate in developed cities and towns. Such as Shanxi Yellow river tributaries is an important trading goods dock, urban residents, and the water quality was $\mathrm{V}$ in 2011.

The water quality of interprovincial water function zone is also affected by hydrodynamic conditions. The water pollution passes from upstream to downstream in the mountain river, which the upstream and downstream is obviously. But in the plain river network region, the flow direction isn't assured, the flow often reveals reversing current. Such as the river in Taihu Lake Basin, the contaminant will flow between upstream and downstream. In other case, one bank of the interprovincial river also won't cause sewage pollutant the water body of the opposite bank, such as in the Yangtze River. But as for small and medium rivers, the pollutants of one shore will contaminate other side bank.

\section{Water ecological compensation methods for provincial water function zone}

If interprovincial water functional zone is polluted, at the first should decide which district has pollution liability, and then estimates the water ecological compensation amount, determined in accordance with pollution liability compensated.

Pollution contributions analysis

Pollution contributions may be determined by the pollutants transformation relationship between the upstream and downstream. For small and medium mixing well river pollutants in cross section, it can be represented by the equation of pollutants transported by the process as Eq.1:

$$
C=C_{0} \exp \left(-K \frac{x}{u}\right)
$$

In the formula (1), $\mathrm{C}_{0}$ is river upstream inflow background concentrations $(\mathrm{mg} / \mathrm{L})$; $\mathrm{u}$ is the flow velocity $(\mathrm{m} / \mathrm{s}) ; K$ is pollutant attenuation coefficient $(1 / \mathrm{d}) ; \mathrm{x}$ is river length from the discharge port to considered cross section $(\mathrm{m})$.

And in wide and shallow river or water body, the equation of pollutants transported by the process as Eq.2:

$$
\mathrm{h}\left(\frac{\partial \mathrm{c}}{\partial \mathrm{t}}+u \frac{\partial c}{\partial x}+v \frac{\partial c}{\partial y}-\frac{\partial}{\partial x} D_{x} \frac{\partial c}{\partial x}-\frac{\partial}{\partial y} D_{y} \frac{\partial c}{\partial y}-\sigma+k c\right)=0
$$

In the formula (2), $h$ is river water depth $(\mathrm{m}) ; \mathrm{u}$ and $\mathrm{v}$ are the flow velocity $(\mathrm{m} / \mathrm{s}) ; k$ is pollutant attenuation coefficient $(1 / \mathrm{d}) ; \mathrm{D}_{\mathrm{x}}$ and $\mathrm{D}_{\mathrm{y}}$ are dispersion coefficient $\left(\mathrm{m}^{2} / \mathrm{s}\right) ; \mathrm{t}$ is time $(\mathrm{s}) . \sigma$ is pollution sources or sinks $(\mathrm{kg} / \mathrm{s})$.

\section{The amount of compensation}

There are many methods to determine the amount of ecological compensation; such as opportunity cost method, cost analysis, the value of water resources law, benefit-sharing coefficient method, ect.

The Li Jinxiu method which is a method for calculate the amount of water ecological compensation is recommended for interprovincial water function zone. Li Jinxiu [17] proposed a hyperbolic function of the relationship between water quality of the environment-social and economic. The function of Li Jinxiu method expression as Eq.3: 


$$
\gamma_{i}=K_{i}\left[\frac{e^{a\left(Q-Q_{T H}\right)}-1}{e^{a\left(Q-Q_{T H}\right)}+1}+M_{i}\right]
$$

In the formula (3), $i$ is an item of economic activity, reflecting the various sub-water pollution economic calculation; $\alpha$ is the characterization of the $S$-curve shape of important parameters, which reflects the water quality of the social and economic impact on the sensitivity level. If $\alpha$ is a larger value, the steeper the function curve, indicating that the socio-economic situation is extremely sensitive to water quality; and if $\alpha$ is a smaller the value, the more level function curve, indicating that the socio-economic situation is less sensitive to water quality. And $\gamma, M, K$ are representing different water quality condition of environmental pollution economic loss. $a$ is a dimensionless coefficients. $Q$ and $Q_{H}$ represent water quality categories, also are dimensionless coefficients.

\section{Compensated objects}

According to the water quality of provincial water function zone, analyze emissions upstream areas, when emissions exceed the total limit their emissions will cause downstream water pollution, excessive water pollution, water quality is not up in this case the upstream area to downstream areas should be compensated, compensated for the downstream water quality objectives nonattainment areas; in order to reduce pollution damage downstream water environment and improve water quality compliance rate, upstream areas for this restrictive measures taken to protect aquatic ecosystems, and adds extra costs, limiting the economic development of the region, this time should be downstream of the upstream areas compensated compensated for the upstream areas.

The relationship of compensating objective and compensated objective might exchange if the level relationship between benefit and protection or damaged objective. The upstream should compensate the downstream if the pollution of upstream worse than given object, and downstream water quality damaged; at the same time, the downstream can compensate the upstream if the pollution of upstream better than given object, and downstream water quality benefited. In other case, as in Taihu basin, compensation body and compensated within the Taihu Lake basin will be affected by the occurrence of a change back and forth flow. The relationship of compensating objective and compensated objective might not clear.

\section{Compensation mode}

Water ecological compensation mode in interprovincial compensation includes economic compensation, compensation policies, financial compensation and material compensation.

Economic compensation includes upstream province pay for downstream province if upstream province have excessive emissions of pollutants which caused downstream water quality poor, and downstream province pay for upstream province if upstream province have protect water quality which benefit for downstream province. The compensation should be determined by the two provinces in accordance with consultations.

Compensation policies include raising taxes on the upstream areas, promoting strict standards for heavily polluting projects, implementing strictly environmental quality inspection, building sewage treatment plants, and preferential policies for compensation refers to tax breaks and other downstream areas. Compensation policy also includes financial compensation as financial transfer payment policy, which transfer finance between the two provinces. The benefit province transfer finance to the damaged province or protecting province. At the same time, incentives also can give to local residents, the local populations who have contribution for the protection of water quality.

Financial compensation includes the establishment of a special fund, sets up special administrative management department. The upstream area should obtain financial compensation when the upstream area to improve water quality, and the economy, ecology of the downstream areas have significantly improved. The downstream area also should obtain financial compensation when the downstream area to improve water quality. Therefore, the funding financial compensation is bidirectional.

Material compensation includes offering food, supplies. We should make sure that the materials are safety and the compensated objectives can obtain compensated materials. 


\section{Conclusions}

The water quality of interprovincial water function zone is relatively low in some basins, with excessive pollution, poor sewage treatment, and imperfect contaminated industrial policies. Water ecological compensation method will help to protect aquatic ecosystems of water functional zones.

Water ecological compensation methods for interprovincial water function zones include determining the compensation targets, the amount and mode of compensation. The practice of water ecological compensation methods in provincial water function zone requires friendly cooperation among provinces, and takes effective program to achieve double-win in interprovincial water function zone water quality objectives.

\section{Acknowledgements}

This study was supported by the IWHR special project (Grant No. 1226), and the National Key Technology R \& D Program (Grant No. 2012BAC06B04).

\section{References}

[1] Li Xiaoyan. Water basin ecological compensation under the Perspective of Ecological Civilization Study (In Chinese) [J]. Rural economy, 2008, (9): 59-62.

[2] Qiao Xuning, Yang Yongju, Yang Degang. Watershed Ecological Compensation Situation and key issue analysis(In Chinese) [J]. Progress in Geography, 2012,31 (4): 395-402.

[3] Zhang Bin. On the water, establish ecological compensation mechanism (In Chinese) [J]. Water Economy, 2010, (1): 58-60.

[4] Liang Yan. Watershed Water Ecological Compensation Mechanism - Case Study of Pearl River Drainage (In Chinese) [C]. Institute of Environmental Science Academic Conference Proceedings excellent Chinese, 2008: 357-360.

[5] Li Jinxiu, Lee Chong, Wu Jian. Financial compensation for water resources protection countermeasures (In Chinese) [J]. Water Resources and Hydropower, 2005, 36 (6): 22-24.

[6] Xu Jian. On the regional water ecological compensation legal coordination mechanisms -

Consideration of ecological compensation Xin'an River Basin (In Chinese) [J]. Legal Forum, 2012,27

(4): 43-50.

[7] Fu Yicheng, Ruan Benqing, Xu Fengran, Chu Limin. Water Yongding River Basin Ecological

Compensation Standards (In Chinese) [J].Hydraulic Engineering, 2012,43 (6): 740-748.

[8]Li Jinxiu, Luo Huihuang, Liao Wengen .Quality excessive risk functional areas to explore (In Chinese) [J]. China Institute of Water Resources and Hydropower Research, 2004,02 (2): 101-103.

[9] The 2011 National Water Resources Bulletin(In Chinese).

[10] 2011 Water resources bulletin(In Chinese).

[11] Provincial boundary water in Haihe River Basin Water Environment Quality Bulletin(In Chinese)

(2011 annual summary).

[12] The 2011 Yellow River Water Resources bulletin(In Chinese).

[13] 2011 Huaihe River Water Resources Journal(In Chinese).

[14] The 2011 Yangtze River Basin water resources in Southwest Gazette(In Chinese).

[15] 2011 Taihu Lake Basin and southeastern rivers water resources bulletin(In Chinese).

[16] 2011 Pearl River Water Resources Bulletin(In Chinese).

[17] Li Jinxiu, Xu Songling . Economic loss for river basin water pollution measurement model(In

Chinese) [J]. Hydraulic Engineering, 2003, (10): 68-74. 